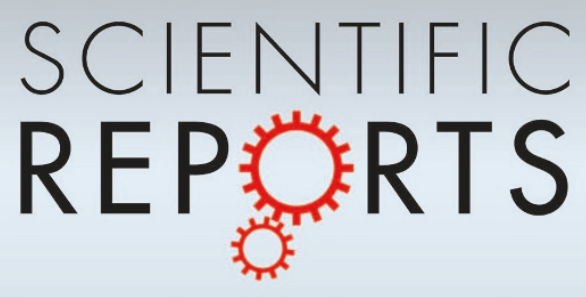

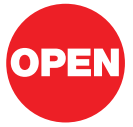

SUBJECT AREAS:

QUANTUM

INFORMATION

QUANTUM MECHANICS

THEORETICAL PHYSICS

INFORMATION THEORY AND COMPUTATION

Received

3 December 2012

Accepted

9 April 2013

Published

20 May 2013

Correspondence and requests for materials should be addressed to

M.Z. (mpzwolak@ gmail.com)

\section{Complementarity of quantum discord and classically accessible information}

\author{
Michael Zwolak' \& Wojciech H. Zurek ${ }^{2}$

\begin{abstract}
'Department of Physics, Oregon State University, Corvallis, OR 97331, ${ }^{2}$ Theoretical Division, MS-B2 13, Los Alamos National Laboratory, Los Alamos, NM 87545 and Santa Fe Institute, Santa Fe, NM 87501.
\end{abstract}

The sum of the Holevo quantity (that bounds the capacity of quantum channels to transmit classical information about an observable) and the quantum discord (a measure of the quantumness of correlations of that observable) yields an observable-independent total given by the quantum mutual information. This split naturally delineates information about quantum systems accessible to observers - information that is redundantly transmitted by the environment - while showing that it is maximized for the quasi-classical pointer observable. Other observables are accessible only via correlations with the pointer observable. We also prove an anti-symmetry property relating accessible information and discord. It shows that information becomes objective - accessible to many observers - only as quantum information is relegated to correlations with the global environment, and, therefore, locally inaccessible. The resulting complementarity explains why, in a quantum Universe, we perceive objective classical reality while flagrantly quantum superpositions are out of reach.

T here is now overwhelming evidence that the Universe we inhabit is made out of quantum "stuff", and therefore quantum to the core. This suggests that we should routinely encounter superpositions. Yet, the world we perceive is resolutely classical. This contrast between quantum expectations and everyday classical reality sets up the problem that puzzled Bohr, Einstein, and many others since the inception of quantum physics ${ }^{1-5}$.

Decoherence ${ }^{6-8}$ changed our view of the quantum-classical correspondence by explaining the stability of pointer states that are selected in the presence of the environment ${ }^{9,10}$. Their nature - in particular, their persistence - made them obvious candidates for "classical states": It was natural to expect that predictably evolving states are good candidates for our everyday "classical reality". Yet, the underlying question - "Why do we, observers, perceive pointer states?" - remains unanswered even after recognizing the role of decoherence in suppressing non-local superpositions. The stability of pointer states fulfills the expectation of predictability built on the daily experience of the classical realm, but it does not address the obvious question: Why is it that observers choose to measure the Universe in a way that reveals pointer states? The key premise of this paper can be summed-up by saying that the choice is made not by observers, but by the medium through which we perceive the Universe.

Quantum Darwinism ${ }^{11-25}$ recognizes that the same environment that is responsible for decoherence serves also as a channel through which information about systems reaches observers, see Fig. 1. We obtain most of our data from the photon environment. The focus of Quantum Darwinism is the redundancy - the presence of multiple copies - of data about certain observables achieved at the expense of the information about complementary observables. Thus, the decohering environment serves not just as a disposal for uncomfortably quantum evidence, but plays a role analogous to a communication channel, an advertising medium in which multiple copies of selected states of the system are present.

Here we show that recognizing the environment as a communication channel is far more than an allegory. Rather, it leads to a precise split of the quantum mutual information between the system and the environment into two components in proportions that depend on the observable of the system: The (maximum) amount of the accessible information about an observable is given by the Holevo quantity that sets an upper limit on the capacity of a quantum channel to transmit classical data ${ }^{26}$. The information that is there in principle, but cannot be found out from the environment alone is given by the quantum discord ${ }^{27-29}$ that characterizes the quantumness of correlations. The Holevo quantity is largest for the pointer observable and decreases for other observables, nearly vanishing for observables that are complementary. Quantum discord makes up the difference between the mutual information (that remains constant) and the Holevo quantity. Thus, under very general conditions this yields a conservation law: While the classically accessible information and quantum discord depend on the observable of the system, their sum does not. 


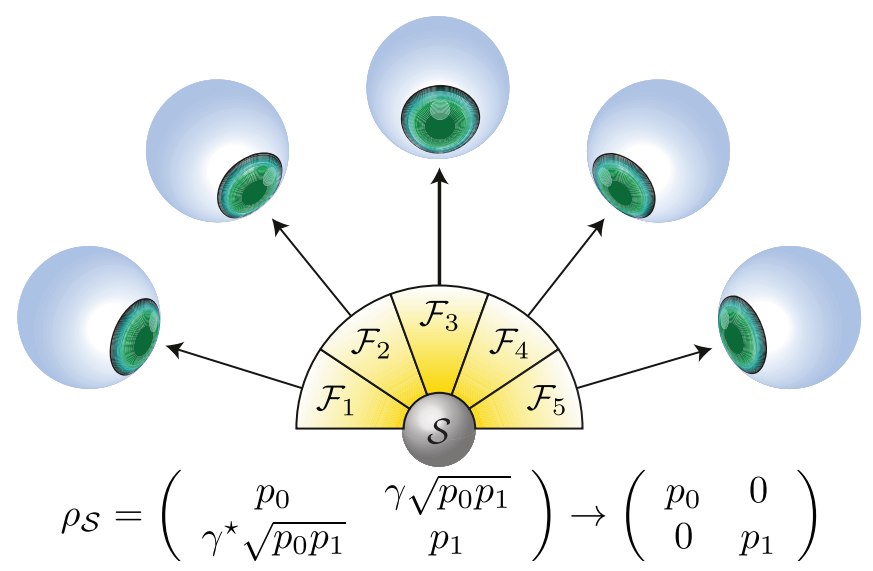

Figure $1 \mid$ The environment as a communication channel. A system, $\mathcal{S}$, interacts with an environment, $\mathcal{E}$, composed of many different fragments, $\mathcal{F}$. While the system decoheres (as indicated by the loss of phase coherence, which is illustrated at the bottom of the figure by the decay of the offdiagonal elements of the density matrix in the pointer basis), the environment fragments each acquire information about $\mathcal{S}$ that can then be transmitted to observers. To learn about the state of the system, each observer intercepts a different fragment.

This division of the mutual information between the Holevo quantity and the quantum discord allows one to understand why the data about the system accessible to observers are effectively limited to the pointer observable. We show that the Holevo quantity for other observables decreases depending on the degree of "misalignment" between them and the eigenstates of the pointer observable. Furthermore, we prove an anti-symmetry relation between discord and the Holevo quantity. This shows that whenever objective, classical information about a system is present, quantum information, as measured by the discord, about this system is out of reach for observers without access to nearly the whole environment and the system - a situation that can occur, at best, only in controlled laboratory experiments.

Observers typically learn about a system of interest, $\mathcal{S}$, indirectly. That is, the environment $\mathcal{E}$ interacts with the system and its fragments acquire information about $\mathcal{S}$. What the fragment $\mathcal{F}$ and $\mathcal{S}$ know about each other is quantified by the mutual information

$$
I(\mathcal{S}: \mathcal{F})=H_{\mathcal{S}}+H_{\mathcal{F}}-H_{\mathcal{S F}},
$$

which measures the total correlations present ${ }^{30}$. Here, $H$ is the von Neumann entropy of the reduced density matrices of $\mathcal{S}, \mathcal{F}$, and $\mathcal{S} \mathcal{F}$. One of the main quantities of interest in Quantum Darwinism is the typical fragment size needed by an observer to learn about the system. That is, what typical fragment size ${ }^{\sharp} \mathcal{F}_{\delta}$ contains $1-\delta$ of the missing information $H_{\mathcal{S}}$ about the system,

$$
I(\mathcal{S}: \mathcal{F}) \geq(1-\delta) H_{\mathcal{S}},
$$

decreasing the remaining entropy to $\delta \cdot H_{\mathcal{S}}$. The parameter $\delta$ is the information deficit, which quantifies the error tolerance of the observers. All entropic quantities that depend on the fragment are averaged with respect to all fragments of the same size, i.e., $I(\mathcal{S}: \mathcal{F})=\langle I(\mathcal{S}: \mathcal{F})\rangle_{\sharp_{\mathcal{F}}}$.

We will, on occasion, use a definition of the fragment size $\sharp \mathcal{F}_{\delta}$ that replaces $I(\mathcal{S}: \mathcal{F})$ in $(2)$ with Holevo quantity $\chi$. We will show, in the case of pointer states, that unless the fragment encompasses almost all of $\mathcal{E}, I(\mathcal{S}: \mathcal{F})$ is essentially equivalent to $\chi$.

When an observer can acquire information about the system from a small fragment of the environment, this means information is redundant - not only can a single observer learn about the system, but many observers can do so independently, and hence objectively. The redundancy is given by

$$
R_{\delta}=\sharp \mathcal{E} /{ }^{\sharp} \mathcal{F}_{\delta} \equiv 1 / f_{\delta},
$$

where $f_{\delta}$ is the fraction size needed to satisfy Eq. (2). The redundancy depends on how the information about $\mathcal{S}$ is stored in $\mathcal{E}$. Obviously, its magnitude is contingent on the information deficit $\delta$ and the total size of the environment, $\sharp \mathcal{E}$. When many environment components independently interact with the system - such as photons with an object in space $-I(\mathcal{S}: \mathcal{F})$ shown in the central plot of Fig. 2 appears as a consequence. The form of this curve indicates the presence of redundant information, as indicated in the figure.

There is a natural connection between Quantum Darwinism and decoherence - in particular, the existence of pointer states. These states survive decoherence with minimal loss of purity and thus "live on" to proliferate information about themselves into the environment.

An operational definition of pointer states (introduced in Refs. 31-33 and known as the "predictability sieve") is based on an intuitive idea: Pointer states become minimally entangled with the environment in the course of decoherence. The predictability sieve criterion is a way to quantify this: For every initial pure state, one measures the entanglement generated dynamically between the system and the environment by computing the entropy or some other measure of predictability from the reduced density matrix of the system. The entropy is a function of time and a functional of the initial state. Pointer states are obtained by minimizing entropy over the initial states and demanding that the answer be robust when varying the decoherence time.

Pointer states are important in determining what information is deposited in the environment. In addition to this connection, the main themes of this work will be how $I(\mathcal{S}: \mathcal{F})$ naturally separates into classical and quantum components, and the implications for the emergence of the classical world.

\section{Results}

The Holevo quantity and discord. We start with a straightforward rewrite of the definition of quantum discord in the setting suitable for Quantum Darwinism: We consider a system $\mathcal{S}$ that is decohered by the environment $\mathcal{E}$. We focus on a fragment $\mathcal{F}$ of $\mathcal{E}$. Quantum discord (from $\mathcal{S}$ to $\mathcal{F}$ ) is then defined as

$$
\mathcal{D}\left(\Pi_{\mathcal{S}}: \mathcal{F}\right)=I(\mathcal{S}: \mathcal{F})-J\left(\Pi_{\mathcal{S}}: \mathcal{F}\right)
$$

for the POVM $\Pi_{\mathcal{S}}$. The asymmetric mutual information $J\left(\Pi_{\mathcal{S}}: \mathcal{F}\right)$ is given by

$$
J\left(\Pi_{\mathcal{S}}: \mathcal{F}\right)=H_{\mathcal{F}}-H\left(\mathcal{F} \mid \Pi_{\mathcal{S}}\right) .
$$

The conditional entropy $H\left(\mathcal{F} \mid \Pi_{\mathcal{S}}\right)$ depends on the density matrices $\rho_{\mathcal{F} \mid s}$ of the fragments $\mathcal{F}$ conditioned on the outcomes $s$ for the POVM. The asymmetric mutual information is equal to $I(\mathcal{S}: \mathcal{F})$ when Bayes' rule relating joint and conditional probabilities holds, as it does for classical systems ${ }^{34}$.

As a result of decoherence the system correlates with the environment. For a two dimensional system,

$$
\left[\alpha|0\rangle_{\mathcal{S}}+\beta|1\rangle_{\mathcal{S}}\right]\left|\psi_{\mathcal{E}}\right\rangle \mapsto \alpha|0\rangle_{\mathcal{S}}\left|\psi_{\mathcal{E} \mid 0}\right\rangle+\beta|1\rangle_{\mathcal{S}}\left|\psi_{\mathcal{E} \mid 1}\right\rangle
$$

where $\left|\psi_{\mathcal{E} \mid 0}\right\rangle$ and $\left|\psi_{\mathcal{E} \mid 1}\right\rangle$ are the conditional states of the environment generated by interaction with the system. The extent of the coherence between 0 and 1 states of the system will depend on the overlap between the corresponding states of $\mathcal{E},\left\langle\psi_{\mathcal{E} \mid 0} \mid \psi_{\mathcal{E} \mid 1}\right\rangle$. In the course of decoherence, the environment also acquires a record of the system's state. Orthogonal conditional states of the environment perfectly record the state of the system. Thus, there is a correspondence between decoherence and the acquisition of a record. As we will see below, in common decoherence scenarios such records are redundant and nearly complete when 0,1 is the pointer basis. 


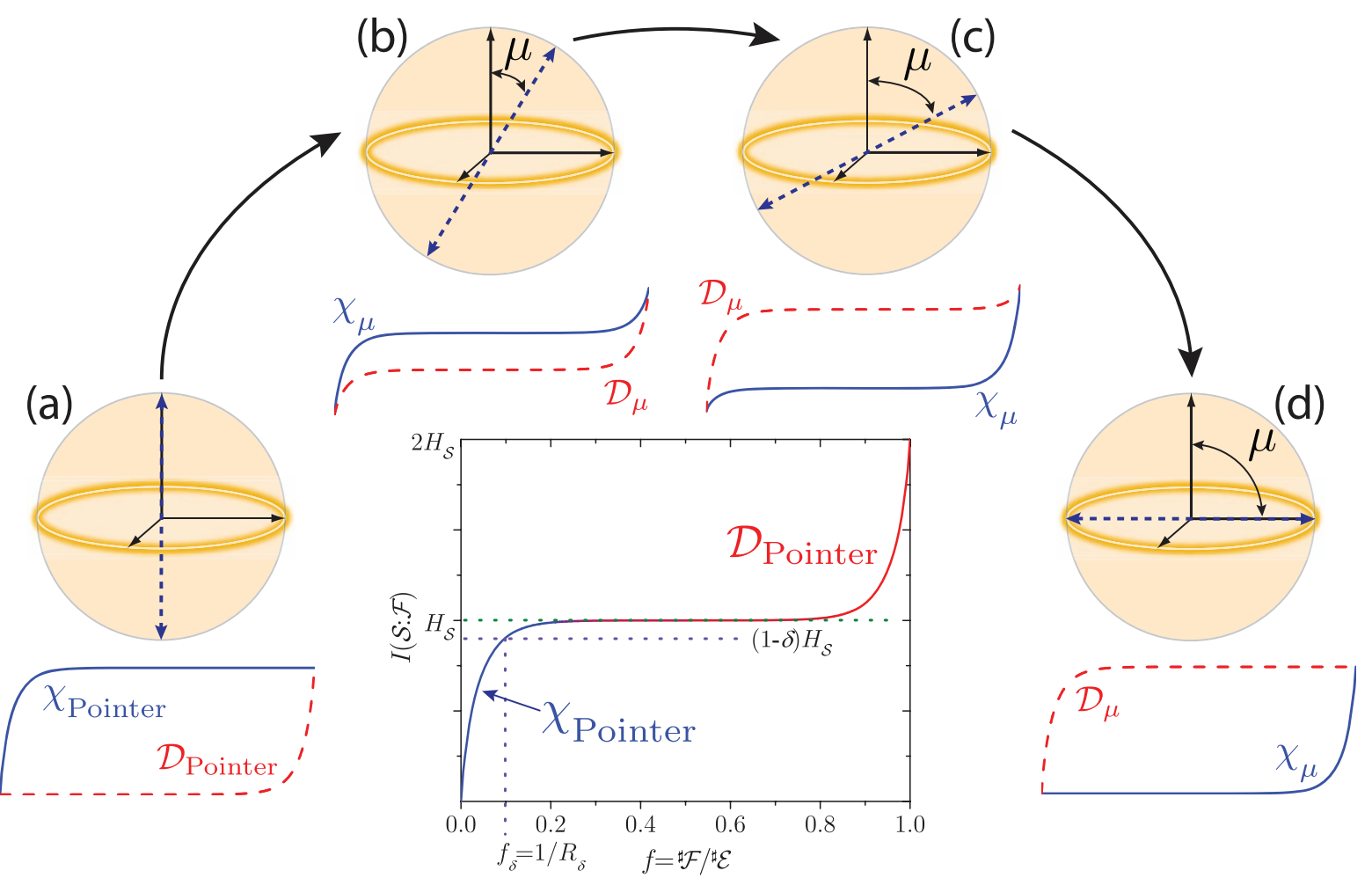

Figure $2 \mid$ Accessible information and quantum discord. The central plot shows the quantum mutual information versus the size of the fragment (given as the fraction $f={ }^{\sharp} \mathcal{F} / \sharp_{\mathcal{E}}$ of the environment) for a pure $\mathcal{S E}$ state, where $\mathcal{E}$ has decohered $\mathcal{S}$. For this and the other figures, we take the system to be twodimensional (spin-1/2 like) and the interaction Hamiltonian to be diagonal in the $\sigma_{z}$ basis (so the eigenstates of $\sigma_{z}$ are "pointer"). (a) The initial steep rise is attributable to $\chi_{\text {Pointer }}$, i.e., classical information about the pointer states of $\mathcal{S}$ being communicated by the environment. Due to the anti-symmetry of the mutual information, the purely quantum information about $\mathcal{S}$ is encoded in global correlations with the environment. (b-d) Rotation by an angle $\mu=\pi / 6$, $2 \pi / 6,3 \pi / 6$ of the observable (i.e., the basis given by $\left|\mu_{+}\right\rangle=\cos (\mu / 2)|0\rangle+\imath \sin (\mu / 2)|1\rangle$ and $\left.\left|\mu_{-}\right\rangle=\sin (\mu / 2)|0\rangle-\imath \cos (\mu / 2)|1\rangle\right)$ will eventually exchange $\chi$ and $\mathcal{D}$. This gradual change between the plot of the Holevo quantity and quantum discord illustrates their complementarity in the setting of the Quantum Darwinism: Information that is locally accessible is maximized for the pointer observable of $\mathcal{S}$. Accessible information about the other observables decreases, and nearly disappears for the complementary observable. Meanwhile, the quantum discord $\mathcal{D}$ increases, so that the sum $\chi+\mathcal{D}$ is constant, independent of the system observable.

Moreover, the environment then acts as a communication channel, broadcasting the record into the larger world.

This is not just an analogy. The asymmetric mutual information in Eq. (5) is the well-known Holevo quantity

$$
\chi\left(\Pi_{\mathcal{S}}: \mathcal{F}\right)=H\left(\sum_{s} p_{s} \rho_{\mathcal{F} \mid s}\right)-\sum_{s} p_{s} H_{\mathcal{F} \mid s},
$$

where $p_{s}$ is the probability of outcome $s$ occurring (see Methods). The Holevo quantity bounds the amount of classical information transmittable over a quantum channel, i.e., the classically accessible information. In the example above, this information is about the states 0 or 1 (more generally, the POVM $\Pi_{\mathcal{S}}$ ). With the recognition that $J$ and $\chi$ are identical, we can now write

$$
I(\mathcal{S}: \mathcal{F})=\chi\left(\Pi_{\mathcal{S}}: \mathcal{F}\right)+\mathcal{D}\left(\Pi_{\mathcal{S}}: \mathcal{F}\right) .
$$

This is the conservation law that we now employ in the discussion of Quantum Darwinism. It is illustrated in Fig. 2. Its salient feature is the fact that its left hand side does not depend on what is of interest to an observer via the set $\Pi_{\mathcal{S}}$, while the ingredients - the classical and quantum components - on the right hand side do.

In the above discussion, we have implicitly assumed that arbitrary POVMs are allowed. In most prior discussions of quantum discord, $\Pi_{\mathcal{S}}$ is usually (although not always) taken as a set of orthogonal states. However, Datta ${ }^{35}$ has shown that quantum discord can always be minimized by using rank one projectors. We accept this generalization to arbitrary POVMs.
In the study of discord there is also a natural temptation to extremize (usually, minimize) $\mathcal{D}\left(\Pi_{\mathcal{S}}: \mathcal{F}\right)$ with respect to the set $\Pi_{\mathcal{S}}$. We are interested in what happens to $\chi$ and $\mathcal{D}$ as the measurement characterized by $\Pi_{\mathcal{S}}$ is varied. This requires a departure from the usual temptations and a consistent - but more general - interpretation of discord.

The sharp division of the whole information present in the $\mathcal{S E}$ correlations into classical, locally accessible $\chi$ and quantum $\mathcal{D}$ (that can be accessed only globally by measurements involving both $\mathcal{S}$ and $\mathcal{E})$ puts immediately to rest the concern that was raised in the early discussions of decoherence ${ }^{36,37}$, where the common criticism was that "one has to ignore the environment to justify the emergence of the pointer observable". Our discussion demonstrates that - by virtue of Holevo's theorem - no measurement of the environment alone can reveal more than $H_{\mathcal{S}}$ - the missing part of the information about the quasi-classical pointer states $\mathcal{S}$. Only global measurement of $\mathcal{S}$ and all of $\mathcal{E}$ can detect phase coherence that is a quantum "leftover" from the phase coherence in the initial state of $\mathcal{S}$.

Thus, classicality that emerges from decoherence does not rest on the assumption of ignoring the environment, but on the realization that the measurements available to the observer are local - that they do not involve global observables with eigenstates that are entangled states of $\mathcal{S}$ and the whole of $\mathcal{E}$. Indeed, even this (already outlandish) requirement does not suffice: To reveal global coherence one would have to measure the state of $\mathcal{S E}$ in such a way that it would not collapse - i.e., one would have to choose a priori a global observable that has that (unknown) entangled state as an eigenstate. A number 
of well-known facts (including, e.g., the no-cloning theorem ${ }^{38,39}$ ) make this impossible.

Anti-symmetry and the emergence of classicality. Let us now analyze the consequences of this split into quantum and classical information. Given an arbitrary rank one POVM and a pure, but otherwise arbitrary, state $\rho_{\mathcal{S E}}$, the quantum discord and the Holevo quantity on complementary fragments of the environment (i.e., $\mathcal{F}$ and the rest of $\mathcal{E}, \mathcal{E} / \mathcal{F}$ ) are related by

$$
\begin{aligned}
\mathcal{D}\left(\Pi_{\mathcal{S}}: \mathcal{E} / \mathcal{F}\right) & =I(\mathcal{S}: \mathcal{E} / \mathcal{F})-\chi\left(\Pi_{\mathcal{S}}: \mathcal{E} / \mathcal{F}\right) \\
& =H_{\mathcal{S}}-H_{\mathcal{S E} / \mathcal{F}}+\sum_{s} p_{s} H_{\mathcal{E} / \mathcal{F} \mid s} \\
& =H_{\mathcal{S}}-H_{\mathcal{F}}+\sum_{s} p_{s} H_{\mathcal{F} \mid s} \\
& =H_{\mathcal{S}}-\chi\left(\Pi_{\mathcal{S}}: \mathcal{F}\right)
\end{aligned}
$$

where we started with Eq. (8). The second to last line used that, for a globally pure state, the conditional state of $\mathcal{E}$ with respect to a rank one POVM element is also pure. The latter implies $H_{\mathcal{E} / \mathcal{F} \mid s}=H_{\mathcal{F} \mid s}$ since $\mathcal{E} / \mathcal{F}$ and $\mathcal{F}$ is a bipartite split of the conditional state of $\mathcal{E}$, just as $H_{\mathcal{S E} / \mathcal{F}}=H_{\mathcal{F}}$.

The relation, Eq. (9), implies that when classical information about $\Pi_{\mathcal{S}}$ is available from small fragments of the environment, $\chi\left(\Pi_{\mathcal{S}}: \mathcal{F}\right) \geq(1-\delta) H_{\mathcal{S}}$, then quantum information is banished to global correlations with the environment, $\mathcal{D}\left(\Pi_{\mathcal{S}}: \mathcal{E} / \mathcal{F}\right) \leq \delta \cdot H_{\mathcal{S}}$. Therefore, local observers - even when they can intercept the rest of the environment, $\mathcal{E} / \mathcal{F}$ - will not have access to it and will never detect superpositions. The reverse is also true for pure $\mathcal{S E}$ states when quantum information is contained in global correlations, then classical information will be present in small fragments of the environment.

It is worth reflecting on this statement further. Figure 3 shows the results of Eq. (9) rewritten to pivot about $H_{\mathcal{S}} / 2$ :

$$
\chi\left(\Pi_{\mathcal{S}}: \mathcal{F}\right)-H_{\mathcal{S}} / 2=H_{\mathcal{S}} / 2-\mathcal{D}\left(\Pi_{\mathcal{S}}: \mathcal{E} / \mathcal{F}\right) .
$$

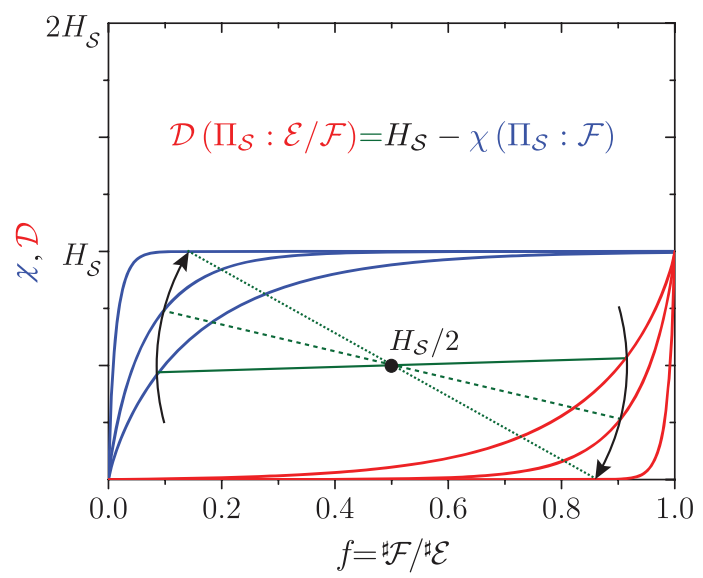

Figure $3 \mid$ Anti-symmetry of discord and the Holevo quantity for a pure $\mathcal{S E}$ state and rank one POVM $\Pi_{\mathcal{S}}$. This symmetry relates discord between $\Pi_{\mathcal{S}}$ and the remainder of the environment $\mathcal{E} / \mathcal{F}$ and the Holevo quantity between $\Pi_{\mathcal{S}}$ and fragment $\mathcal{F}$. As the Holevo quantity increases - i.e., as the classical information transmitted by the environment increases - the discord on the opposite side of the axis decreases. Since the discord is monotonically increasing with $f$, this means that the quantum information about $\mathcal{S}$ is pushed into correlations with the global environment as redundant (classical) information is increased. Thus, a state which has redundant information will have quantum information encoded in the environment, implying that Quantum Darwinism is in action.
This shows that increasing the redundant (classical) information stored in a small fragment $\mathcal{F}$ decreases the quantum information in the much larger fragment $\mathcal{E} / \mathcal{F}$. In other words, a world where objective information is present is also a world with quantum information inaccessible to all but the most encompassing observer. The loss or inaccessibility of any tiny component of the environment will preclude the retrieval of this quantum information.

For arbitrary $\mathcal{S E}$ states, including mixed states, Eq. (9) is replaced by the inequality (see Methods)

$$
\mathcal{D}\left(\Pi_{\mathcal{S}}: \mathcal{E} / \mathcal{F}\right) \leq H_{\mathcal{S}}-\chi\left(\Pi_{\mathcal{S}}: \mathcal{F}\right) .
$$

Thus, it can be stated unequivocally that whenever redundant information is present, quantum information is relegated to global correlations with the environment. We can, as well, examine what happens in particular mixed states. If only $\mathcal{S}$ is mixed, for instance, the discord will just be further suppressed - not only is it contained solely in global correlations, but it can be totally absent (see, e.g., Ref. 21 ). In the cases studied in Refs. 18,21 , the initial mixedness of $\mathcal{E}$ reduced the redundancy but quantum information stayed banished to global correlations.

Branching states and surplus decoherence. To "set the baseline" for physical application of these results, we simplify the rest of our study to the two scenarios that accurately approximate commonly encountered situations - the emergence of branching states via decoherence ${ }^{13,14,40}$ and what we will call "surplus decoherence". Branching happens when the evolution of the decohering system does not create transitions between its pointer states, and the imprints of these states on the environment components are unaffected by the evolution of the remaining $\mathcal{E}$. This is a good approximation of what happens when the photon environment scatters from a heavy object: Subsystems of the environment (photons) individually interact with the system, each pushing $\mathcal{S}$ closer to a localization, but they do not interact with each other ${ }^{22,24}$. Fragments $\mathcal{F}$ consist of collection of such subsystems. On a more formal level, the situation will give rise to Eq. (6) where $\left|\psi_{\mathcal{E} \mid s}\right\rangle=\otimes_{k}\left|\psi_{k \mid s}\right\rangle$. Branching states can also occur approximately, at times shorter than the dissipation timescale ${ }^{15}$ or the mixing timescale of environment components ${ }^{25}$.

Surplus decoherence occurs when a part of $\mathcal{E}$ suffices to decohere $\mathcal{S}$ - i.e., when both the environment $\mathcal{E}$ and the environment without some fragment $\mathcal{E} / \mathcal{F}$ completely decohere the system. In other words, the environment is so large that the state of the system and a fragment will have the form

$$
\rho_{\mathcal{S F}}=\sum_{\hat{s}} p_{\hat{s}}|\hat{s}\rangle\langle\hat{s}| \otimes \rho_{\mathcal{F} \mid \hat{s}}
$$

where $\rho_{\mathcal{F} \mid \hat{s}}$ is the conditional state of the fragment given the state $\hat{s}$. That is, there is some orthogonal basis $\hat{\Pi}_{\mathcal{S}}$ of the system - the pointer basis - such that $\mathcal{E} / \mathcal{F}$ will decohere even the joint state of $\mathcal{S F}$ giving a discord-free form. In many situations (but not always) branching will give rise to surplus decoherence. Together, these two cases accurately approximate commonly encountered system-fragment states generated by decoherence. Thus, it is enlightening to study what are the consequences of these conditions for the division of the mutual information into classical and and quantum components.

For the final part of the paper, we will mostly make use of pure, branched $\mathcal{S E}$ states, which have the form

$$
\left|\psi_{\mathcal{S}}\right\rangle\left[\bigotimes_{k \in \mathcal{E}}\left|\psi_{k}\right\rangle\right] \mapsto \sum_{\hat{s}} c_{\hat{s}}|\hat{\boldsymbol{s}}\rangle\left[\bigotimes_{k \in \mathcal{E}}\left|\psi_{k \mid \hat{s}}\right\rangle\right],
$$

where $\hat{s}$ indicates a pointer state. For these states, $I(\mathcal{S}: \mathcal{F})$ has the shape characteristic of Quantum Darwinism, see Fig. 2: It raises steeply from $I(\mathcal{S}: \mathcal{F})=0$ at $\sharp \mathcal{F}=0$, the size of $\mathcal{F}$, to a plateau of $H_{\mathcal{S}}$ - to the level of the entropy of the system. This plateau is in many 
ways the dominant feature of $I(\mathcal{S}: \mathcal{F})$ that arises in Quantum Darwinism.

Pointer states minimize discord. When $I(\mathcal{S}: \mathcal{F})$ is decomposed into $\chi$ and $\mathcal{D}$, the initial rise is attributable to the channel capacity $\chi$ that reveals the state of the pointer basis. The mutual information continues then at the level $H_{\mathcal{S}}$ until $\sharp \mathcal{F} \simeq \sharp_{\mathcal{E}}$ where the fragment becomes so large that it encompasses essentially all of the environment. For a state of the form in Eq. (13), the discord is

$$
\begin{aligned}
\mathcal{D}\left(\Pi_{\mathcal{S}}: \mathcal{F}\right) & =H_{\mathcal{S}}-H_{\mathcal{S F}}+\sum_{s} p_{s} H_{\mathcal{F} \mid s} \\
& =\mathcal{D}\left(\hat{\Pi}_{\mathcal{S}}: \mathcal{F}\right)+\sum_{s} p_{s} H_{\mathcal{F} \mid s},
\end{aligned}
$$

where the second line follows from the fact that the states of $\mathcal{F}$ conditional on a pointer state on $\mathcal{S}$ are pure (just a pure $\mathcal{E}$ state will suffice to ensure the conditional state on $\mathcal{F}, \rho_{\mathcal{F} \mid \hat{s}}$, is pure so $H\left(\mathcal{F} \mid \Pi_{\mathcal{S}}\right)$ in Eq. (5) vanishes). The second term in Eq. (14) is positive but zero for the pointer basis and thus the pointer basis minimizes the discord. Using the conservation law, when the discord is minimized, the Holevo quantity is maximized. Therefore, the maximum accessible quantum information is largest for the pointer basis.

Moreover, for branched states, including ones evolved from initially mixed states of $\mathcal{S E}$, the discord is given by $\mathcal{D}\left(\hat{\Pi}_{\mathcal{S}}: \mathcal{F}\right)$ $=H_{\mathcal{S} d \mathcal{E}}-H_{\mathcal{S} d \mathcal{E} / \mathcal{F}}$, i.e., a difference of the system entropy decohered by the full environment $\mathcal{E}$ and by the remainder of the environment $\mathcal{E} / \mathcal{F}$ (see Eq. (17) of Ref. 21). For initially mixed states, the pointer basis will not necessarily minimize the discord. However, the discord with respect to the pointer basis is exponentially small in the size of the environment, and we have the state approximately given by Eq. (12). Thus, for all practical purposes, one has

$$
I(\mathcal{S}: \mathcal{F})=\chi\left(\hat{\Pi}_{\mathcal{S}}: \mathcal{F}\right)
$$

This equality is equivalent to the assumption of surplus decoherence. While the surplus decoherence condition can be framed independently of the dynamics/Hamiltonian, we know that the physically relevant scenario of many environment components interacting independently with the system (e.g., the photon environment) leads naturally to surplus decoherence. If one removed a small fragment of the photon environment, systems would still be rapidly decohered. In fact, in this scenario, Eq. (12) is true up to corrections $\mathcal{O}\left(\Pi_{k \in \mathcal{E} / \mathcal{F}} d_{k}\right)$, where $\left|d_{k}\right|<1$ is the decoherence factor from environment component $k$. Yet, the state resulting from surplus decoherence has a deep implication: The mutual information between $\mathcal{S}$ and $\mathcal{F}$ contains only classical information about the pointer basis, $I(\mathcal{S}: \mathcal{F})=$ $\chi\left(\hat{\Pi}_{\mathcal{S}}: \mathcal{F}\right)$.

For a pure state of $\mathcal{S}$ and $\mathcal{E}$, the resulting $I(\mathcal{S}: \mathcal{F})$ is antisymmetric with respect to $H_{\mathcal{S}}$ and $\sharp \mathcal{F}=\sharp \mathcal{E} / 2$. This means that the initial rise to the plateau must be matched by the steep rise from the plateau to $I(\mathcal{S}: \mathcal{F} \rightarrow \mathcal{E})=2 H_{\mathcal{S}}$ as the size of $\mathcal{F}$ approaches the size of the whole of $\mathcal{E}$. This final rise is caused by the rapid increase of $\mathcal{D}\left(\hat{\Pi}_{\mathcal{S}}: \mathcal{F}\right)$ from 0 to $H_{\mathcal{S}}$.

The physical implications of these runs of $\chi$ and $\mathcal{D}$ are straightforward and appealing: The information that can be obtained from the environment about the pointer observable of $\mathcal{S}$ by measurement of a fragment $\mathcal{F}$ quickly saturates to the value set by its entropy $H_{\mathcal{S}}$. Moreover, only $\chi$ of information can be obtained from $\mathcal{E}$ no matter how large is the fragment $\mathcal{F}$. This follows from Holevo's theorem ${ }^{26}$ : $\chi$ is an upper bound on the information that can be extracted from the quantum channel. The rapid rise of $I(\mathcal{S}: \mathcal{F})$ at the very end is then completely due to discord - due to the quantum information that can be accessed only via global measurements that involve both $\mathcal{S}$ and $\mathcal{E}$.

This consideration implies that when many environment components interact independently with the system, only classical information will be transferred into the environment: There is a basis chosen by the environment's interaction with the system that is proliferated into the environment. In this sense, the generation of branching states will always proliferate information about the pointer basis. Incidentally, this is the world in which we live, where photons interact independently with systems, proliferating redundant - and therefore objective - information and conveniently hiding quantum information. We can take this latter step forward, and show that one can only - in a way that will be clear in a moment - find out about the pointer states.

Only pointer states can be redundant. We now consider an attempt to extract, from fragments of $\mathcal{E}$, information about $\sigma_{y}$, which is complementary to the pointer observable $\sigma_{z}$ (see Fig. 2). Success would imply detection of evidence of quantumness - catching Schrödinger's cat in a superposition of dead and alive. The plot of quantum mutual information $I(\mathcal{S}: \mathcal{F})$ is, of course, independent of the observable of $\mathcal{S}$. The two contributions, though, "change places". Now it is the discord that raises rapidly (a feature that we address below), its graph matching the plot of $\chi$ in the previous pointer observable case. By contrast, $\chi$ remains close to zero until the very end, where measurement of all of $\mathcal{E}$ could in principle reveal the eigenstate of $\sigma_{y}$ with which $\mathcal{E}$ is entangled. Still, there is no information about $\sigma_{y}$ that can be gleaned from any fraction of $\mathcal{E}$ the whole of $\mathcal{E}$ is needed to get $\sigma_{y}$ (if it is available at all).

An intermediate case - spin at an angle $\mu$ from $\sigma_{z}$-is an obvious next case to consider. Now the plateau of the corresponding $\chi_{\mu}=\chi\left(\Pi_{\mathcal{S}}: \mathcal{F}\right)$ is lower than the missing information about the "intermediate" observable, $H\left(\Pi_{\mathcal{S}}\right)$. Under surplus decoherence and when $\mathcal{F}$ holds a perfect record of the pointer states, $\chi$ for another observable is given by

$$
\chi\left(\Pi_{\mathcal{S}}: \mathcal{F}\right)=H\left(\Pi_{\mathcal{S}}\right)-H\left(\Pi_{\mathcal{S}} \mid \hat{\Pi}_{\mathcal{S}}\right) .
$$

Figure 4 shows the Holevo quantity versus the fragment size and the angle for the example system under consideration. When $H\left(\Pi_{\mathcal{S}} \mid \hat{\Pi}_{\mathcal{S}}\right)>\delta \cdot H\left(\Pi_{\mathcal{S}}\right)$, redundant records of the observable $\Pi_{\mathcal{S}}$ can not exist - at most "there can be only one" copy. When $\delta$ is small, but non-zero, only states that are very nearly the pointer states can be determined. An equivalent result to Eq. (16) was obtained in Ref. 12. We show in the Methods how to extend it to imperfect records.

The Holevo quantity for pointer $\hat{\Pi}_{\mathcal{S}}$ is the discord for a complementary $\Pi_{\mathcal{S}}$. Continuing from the above results, as $\chi_{\mu}$ is reduced, $\mathcal{D}_{\mu}$ must make up the difference due to the conservation law. In case of surplus decoherence, this means

$$
\chi\left(\hat{\Pi}_{\mathcal{S}}: \mathcal{F}\right)=\chi\left(\Pi_{\mathcal{S}}: \mathcal{F}\right)+\mathcal{D}\left(\Pi_{\mathcal{S}}: \mathcal{F}\right) .
$$

When the basis $\Pi_{\mathcal{S}}$ is complementary to the pointer basis $\hat{\Pi}_{\mathcal{S}}$ the plateau in the Holevo quantity is zero, and the accessible information about the pointer basis turns into the complementary discord. We define a complementary POVM $\Pi_{\mathcal{S}}$ as one where all elements satisfy

$$
\left\langle\hat{s}\left|\pi_{s}\right| \hat{s}\right\rangle=q_{s},
$$

where $q_{s}$ is independent of the pointer state $\hat{s}$. That is, each $\pi_{s}$ is unbiased with respect to any pointer state $|\hat{\boldsymbol{s}}\rangle$. An example would be the $\sigma_{y}$ basis when $\sigma_{z}$ is pointer.

Assuming a state of the form in Eq. (12), the conditional state with respect to the outcome $s$ is

$$
\begin{aligned}
p_{s} \rho_{\mathcal{F} \mid s} & =\operatorname{tr}_{\mathcal{S}} \sqrt{\pi_{s}} \rho_{\mathcal{S F}} \sqrt{\pi_{s}} \\
& =q_{s} \rho_{\mathcal{F}},
\end{aligned}
$$

yielding $p_{s}=q_{s}$. In other words, the conditional states are just the reduced states of $\mathcal{F}$. Then, however, 
(a)

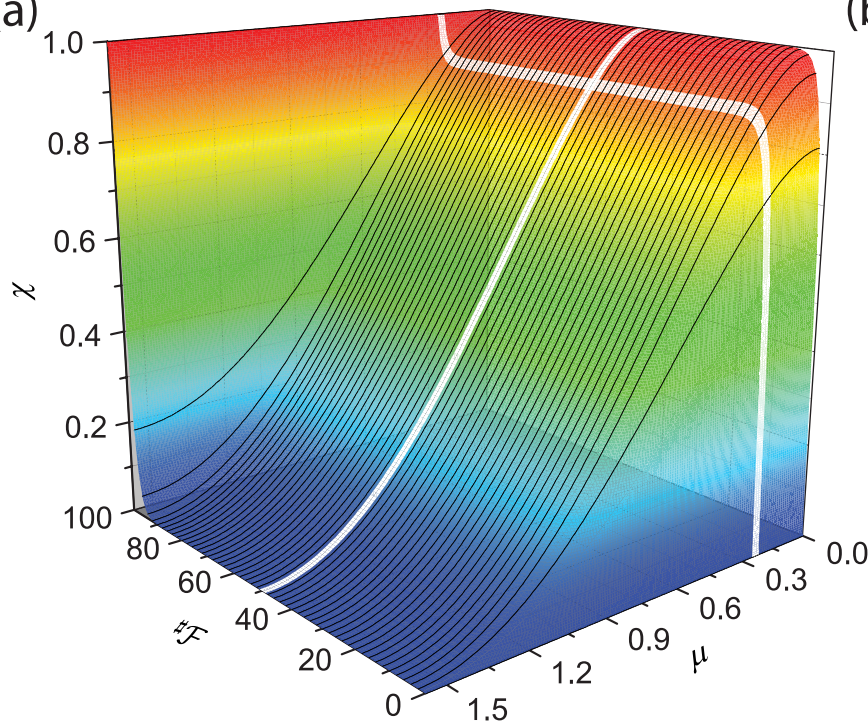

(b)

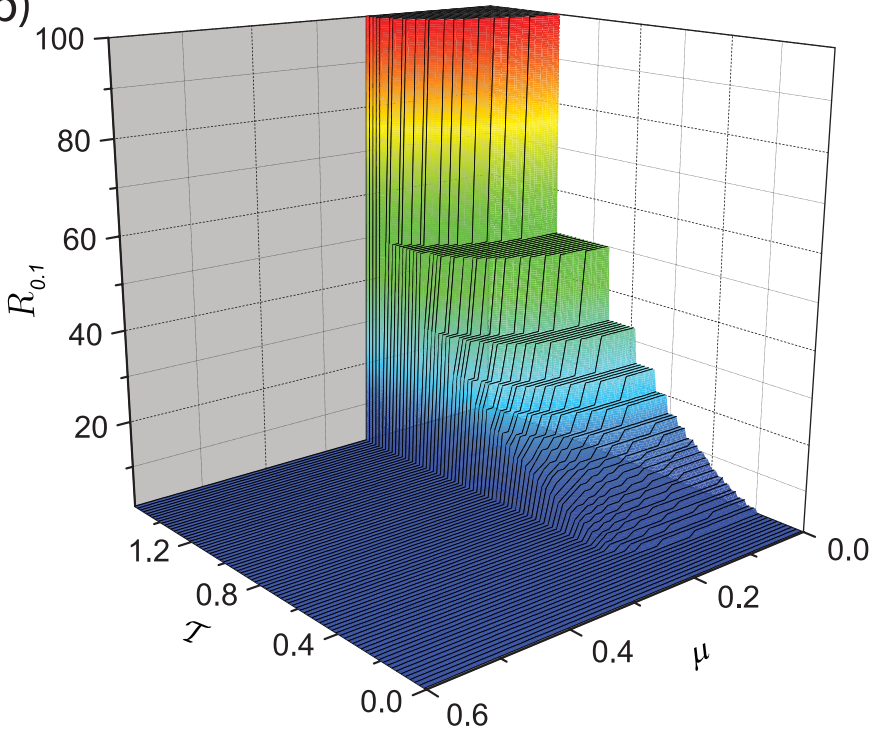

Figure $4 \mid$ Observable dependence of the classical information and its redundancy. (a) Plot of the Holevo quantity versus basis (defined by the angle $\mu$ ) and fragment size for the representative spin model. Here, the off-diagonal elements of the reduced state of the system, $\rho_{\mathcal{S}}$, are suppressed by the decoherence factor $(\cos \mathcal{T})^{\sharp_{\mathcal{E}}}$, where $\mathcal{T}$ is the action (in units of $h$ ) that results from the coupling of a single environment spin to the system. The plot shows the Holevo quantity for $\mathcal{T}=\pi / 2, \sharp \mathcal{E}=100$, and a symmetric and pure environment state. The white line parallel to the fragment size axis demarcates where redundancy is no longer possible for $\delta=0.1$. The other white line plots Eq. (16) for the plateau value of $\chi$. Note that the discord versus basis would be a similar figure, but larger on the side $\mu=\pi / 2$, as it is given by $\mathcal{D}\left(\Pi_{\mathcal{S}}: \mathcal{F}\right)=I(\mathcal{S}: \mathcal{F})-\chi\left(\Pi_{\mathcal{S}}: \mathcal{F}\right)$. (b) Redundancy versus $\mu$ and $\mathcal{T}$ computed by finding $\forall_{\mathcal{F}}$ such that $\chi\left(\Pi_{\mathcal{S}}: \mathcal{F}\right) \geq H\left(\Pi_{\mathcal{S}}\right)(1-\delta)$. Compared to Eq. (2), this calculation of $\sharp_{\mathcal{F}}$ drops terms that are exponentially small. For the example case here, the missing information is $H\left(\Pi_{\mathcal{S}}\right)=H\left(p_{\mu}\right)=1$, with $H\left(p_{\mu}\right)$ the binary entropy of the new probability distribution. The probabilities of detecting the spin in the " $+/-$ " directions along the axis defined by $\mu$ are $p_{+}=p_{0} \cos ^{2}[\mu / 2]+p_{1} \sin ^{2}[\mu / 2]$ and $p_{-}=1-p_{+}$, where $p_{0,1}=1 / 2$ are the probabilities of the pointer states occurring. The conditional entropy is given by $H\left(\Pi_{\mathcal{S}} \mid \hat{\Pi}_{\mathcal{S}}\right)=H\left(\cos ^{2}[\mu / 2]\right)$.

$$
\begin{aligned}
\mathcal{D}\left(\Pi_{\mathcal{S}}: \mathcal{F}\right) & =H_{\mathcal{S}}-H_{\mathcal{S F}}+\sum_{s} p_{s} H_{\mathcal{F} \mid s} \\
& =H_{\mathcal{S}}-\left(H_{\mathcal{S}}+\sum_{\hat{s}} p_{\hat{s}} H_{\mathcal{F} \mid \hat{s}}\right)+\sum_{s} p_{s} H_{\mathcal{F}} \\
& =H_{\mathcal{F}}-\sum_{\hat{s}} p_{\hat{s}} H_{\mathcal{F} \mid \hat{s}} \\
& =\chi\left(\hat{\Pi}_{\mathcal{S}}: \mathcal{F}\right)
\end{aligned}
$$

where the second line follows from the surplus decoherence condition and also Eq. (19). Thus, the plot of $\mathcal{D}\left(\Pi_{\mathcal{S}}: \mathcal{F}\right)$ will follow that of $\chi\left(\hat{\Pi}_{\mathcal{S}}: \mathcal{F}\right)$. When one does not exactly have surplus decoherence, the corrections will depend on the magnitude of the off-diagonal elements in $\rho_{\mathcal{S F}}$. For many environment components independently decohering the system these elements become exponentially small in the environment size (exponentially small in ${ }^{\sharp} \mathcal{E}-{ }^{\sharp} \mathcal{F}$ ).

\section{Discussion}

We have demonstrated that the quantum mutual information naturally separates into classical and quantum components. We proved that a world containing redundant information about a system necessarily implies that quantum information is inaccessible. In other words, when information about some basis has been proliferated into the environment (making it "objective"), then the quantum information - information about superpositions of that basis (e.g., of the initial state of the system) - is suppressed. This information cannot be obtained by observers intercepting only a fragment of the environment. Rather, they need the whole environment and the system to retrieve it. Furthermore, the information proliferated into the environment is not destroyed by further change/monitoring of the system. Once the system has deposited multiple copies of its information, they are "here to stay".
Although discord is often studied these days, questions remain about its fundamental significance. This work suggests that discord has a robust role to play in defining what quantum information means. We have explored it in a specific context of the quantumto-classical transition in the setting of Quantum Darwinism, but some of our conclusions are clearly relevant more generally ${ }^{41}$. Our results also help draw a distinction between the more flagrant aspects of the "quantumness of correlations" captured by entanglement and the quantumness of correlations that are separable - devoid of entanglement - and yet not completely classical.

\section{Methods}

The Holevo quantity and discord. The quantum discord (from $\mathcal{S}$ to $\mathcal{F}$ ) is

$$
\mathcal{D}\left(\Pi_{\mathcal{S}}: \mathcal{F}\right)=I(\mathcal{S}: \mathcal{F})-J\left(\Pi_{\mathcal{S}}: \mathcal{F}\right)
$$

given the POVM $\Pi_{\mathcal{S}}, \sum_{s} \Pi_{s}=\mathbf{I}$, where $\pi_{s}$ are the elements of $\Pi_{\mathcal{S}}$. The quantum mutual information is $I(\mathcal{S}: \mathcal{F})=H_{\mathcal{S}}+H_{\mathcal{F}}-H_{\mathcal{S F}}$, where $H$ is the von Neumann entropy of the of the density matrices $\rho_{\mathcal{S}}, \rho_{\mathcal{F}}$, and $\rho_{\mathcal{S F}}$ that one obtains by tracing out "the rest", i.e., $\rho_{\mathcal{S}}=\operatorname{tr}_{\mathcal{E}} \rho_{\mathcal{S E}}, \rho_{\mathcal{F}}=\operatorname{tr}_{\mathcal{S E} / \mathcal{F}} \rho_{\mathcal{S E}}, \ldots$ All entropic quantities are averaged with respect to all fragments of the same size, e.g., $I(\mathcal{S}: \mathcal{F})=\langle I(\mathcal{S}: \mathcal{F})\rangle_{\sharp \mathcal{F}}$. Most results hold without averaging, and it will be stated when averaging is necessary.

The state of $\mathcal{F}$ conditional on outcome $s$ on $\mathcal{S}$ is

$$
p_{s} \rho_{\mathcal{F} \mid s}=\operatorname{tr}_{\mathcal{S}} \sqrt{\pi_{s}} \rho_{\mathcal{S F}} \sqrt{\pi_{s}},
$$

where $p_{s}$ is the probability of obtaining outcome $s$. Denoting the entropy of $\mathcal{F}$ given outcome $s$ as $H_{\mathcal{F} \mid s}=-\operatorname{tr} \rho_{\mathcal{F} \mid s} \log \rho_{\mathcal{F} \mid s}$, the asymmetric mutual information is

$$
\begin{aligned}
J\left(\Pi_{\mathcal{S}}: \mathcal{F}\right) & =H_{\mathcal{F}}-H\left(\mathcal{F} \mid \Pi_{\mathcal{S}}\right) \\
& =H_{\mathcal{F}}-\sum_{s} p_{s} H_{\mathcal{F} \mid s} . \\
& =H\left(\sum_{s} p_{s} \rho_{\mathcal{F} \mid s}\right)-\sum_{s} p_{s} H_{\mathcal{F} \mid s} .
\end{aligned}
$$

The last line used 


$$
\begin{aligned}
\sum_{s} p_{s} \rho_{\mathcal{F} \mid s} & =\sum_{s} \operatorname{tr}_{\mathcal{S}} \sqrt{\pi_{s}} \rho_{\mathcal{S F}} \sqrt{\pi_{s}} \\
& =\sum_{s} \operatorname{tr}_{\mathcal{S}} \pi_{s} \rho_{\mathcal{S F}}=\rho_{\mathcal{F}},
\end{aligned}
$$

which follows from the cyclic property of the partial trace (with the identity acting on $\mathcal{F}$ ). Even though the POVM does not uniquely set the post-measurement state of $\mathcal{S}$, it will for $\mathcal{F}$. Equation (22) is of course the equation for the Holevo quantity $\chi$, which we denote as $\chi\left(\Pi_{\mathcal{S}}: \mathcal{F}\right)$ since it depends on the POVM $\Pi_{\mathcal{S}}$. The similarity with the Holevo quantity was also noticed in Ref. 29.

Anti-symmetry and the emergence of classicality. Assuming a pure $\mathcal{S E}$ state, the mutual information, $\langle I(\mathcal{S}: \mathcal{F})\rangle_{\mathcal{H}_{\mathcal{F}}}$ (designated by $I(\mathcal{S}: \mathcal{F})$ ), is antisymmetric about the point ${ }^{\sharp} \mathcal{F}={ }^{\sharp} \mathcal{E} / 2$ (see Ref.14). That is,

$$
I(\mathcal{S}: \mathcal{F})=2 H_{\mathcal{S}}-I(\mathcal{S}: \mathcal{E} / \mathcal{F})
$$

and $I(\mathcal{S}: \mathcal{E} / 2)=H_{\mathcal{S}}$. This is shown readily by writing out the mutual information

$$
\begin{aligned}
I(\mathcal{S}: \mathcal{F})+I(\mathcal{S}: \mathcal{E} / \mathcal{F}) & =H_{\mathcal{S}}+H_{\mathcal{F}}-H_{\mathcal{S} \mathcal{F}}+H_{\mathcal{S}}+H_{\mathcal{E} / \mathcal{F}}-H_{\mathcal{S E} / \mathcal{F}} \\
& =H_{\mathcal{S}}+H_{\mathcal{F}}-H_{\mathcal{E} / \mathcal{F}}+H_{\mathcal{S}}+H_{\mathcal{E} / \mathcal{F}}-H_{\mathcal{F}} \\
& =H_{\mathcal{S}}+H_{\mathcal{S}} \\
& =H_{\mathcal{S}}+H_{\mathcal{E}}-0=I(\mathcal{S}: \mathcal{E})
\end{aligned}
$$

where we used that $\mathcal{S E}$ is in a pure state to relate entropies across a bipartite split (e.g., $H_{\mathcal{S F}}=H_{\mathcal{E} / \mathcal{F}}$. Equation (24) holds regardless of whether averaging is done. However, only when the mutual information refers to the average of all fragments of a given size does it imply that the mutual information versus ${ }^{\sharp} \mathcal{F}$ is antisymmetric about its midpoint (at ${ }^{\sharp} \mathcal{F}={ }^{\sharp} \mathcal{E} / 2, I(\mathcal{S}: \mathcal{F})=H_{\mathcal{S}}$ from Eq. (24) when averaged). This is easy to see by examining, e.g., the mutual information in the state

$$
\left(|00\rangle_{\mathcal{S F}_{1}}+|11\rangle_{\mathcal{S F}_{1}}\right)|0 \cdots 0\rangle_{\mathcal{E} / \mathcal{F}_{1}}
$$

where only a single environment spin is correlated with the system. If ordering is maintained in the environment spins, the mutual information is clearly not antisymmetric, even though Eq. (24) holds. It becomes so only when averaging is performed and the mutual information then depends on the (typical) fragment size only.

In the main text, we derived a stronger result

$$
\mathcal{D}\left(\Pi_{\mathcal{S}}: \mathcal{E} / \mathcal{F}\right)=H_{\mathcal{S}}-\chi\left(\Pi_{\mathcal{S}}: \mathcal{F}\right)
$$

for an arbitrary rank one $\mathrm{POVM}_{\mathcal{S}}$ and a pure state of $\mathcal{S E}$. Averaging is not required for Eq. (26). More generally,

$$
\mathcal{D}\left(\Pi_{\mathcal{S}}: \mathcal{E} / \mathcal{F}\right) \leq H_{\mathcal{S}}-\chi\left(\Pi_{\mathcal{S}}: \mathcal{F}\right)
$$

for an arbitrary rank one POVM $\Pi_{\mathcal{S}}$ and arbitrary, potentially mixed, state of $\mathcal{S E}$. To show this, consider any state $\rho_{\mathcal{S E}}$ and purify the state to $\left|\psi_{\mathcal{S E E}}\right\rangle\left\langle\psi_{\mathcal{S E E}}\right|$. Starting with the conservation law,

$$
\begin{aligned}
\mathcal{D}\left(\Pi_{\mathcal{S}}: \mathcal{E} / \mathcal{F}\right) & =H_{\mathcal{S}}-H_{\mathcal{S E} / \mathcal{F}}+\sum_{s} p_{s} H_{\mathcal{E} / \mathcal{F} \mid s} \\
& =H_{\mathcal{S}}-H_{\mathcal{F E}}+\sum_{s} p_{s} H_{\mathcal{F} \mathcal{E}^{\prime} \mid s} \\
& =H_{\mathcal{S}}-\chi\left(\Pi_{\mathcal{S}}: \mathcal{F} \mathcal{E}^{\prime}\right)
\end{aligned}
$$

where we used that for a rank one POVM the conditional state of $\mathcal{E} \mathcal{E}^{\prime}$ is pure, and therefore $H_{\mathcal{E} / \mathcal{F} \mid s}=H_{\mathcal{F E} \mid s}$. From the data processing inequality ${ }^{42}$, if we trace out the purifying environment $\mathcal{E}^{\prime}$, then this reduces the Holevo quantity

$$
\chi\left(\Pi_{\mathcal{S}}: \mathcal{F}\right) \leq \chi\left(\Pi_{\mathcal{S}}: \mathcal{F} \mathcal{E}^{\prime}\right) .
$$

and gives Eq. (27). We note that, in addition to pure states, there are cases of interest here where equality holds in Eq. (28) (i.e., when $\mathcal{F}$ is mixed but only within disjoint subspaces that are correlated with $\mathcal{S}$ ).

Only pointer states can be redundant. We have seen that the pointer states minimize discord for pure, branching states (and for surplus decoherence), and that branching states lead to surplus decoherence up to exponentially small corrections. Let's see what happens to transmitted information for surplus decoherence. Consider the case where a perfect record of the pointer basis exist in a fragment of the environment,

$$
\chi\left(\hat{\Pi}_{\mathcal{S}}: \mathcal{F}\right)=H_{\mathcal{S}},
$$

i.e., suppose that the conditional states $\rho_{\mathcal{F} \mid \hat{s}}$ are orthogonal for the PVM $\hat{\Pi}_{\mathcal{S}}$. Now consider a POVM $\Pi_{\mathcal{S}}$, that we can define by the quantities

$$
p_{s \hat{s}}=\left\langle\hat{s}\left|\pi_{s}\right| \hat{s}\right\rangle,
$$

which give the conditional probabilities for $s$ to occur given $\hat{s}$. For the state $\rho_{\mathcal{S F}}$ in Eq. (12), the probability of outcome $s$ is

$$
p_{s}=\operatorname{tr}_{\mathcal{S F}} \pi_{s} \rho_{\mathcal{S F}}=\sum_{s} p_{s s} p_{\hat{s}} .
$$

Further, since the $p_{s \hat{s}}$ are conditional probabilities, they obey

$$
\sum_{s} p_{s \hat{s}}=1
$$

which is readily obtained by using that $\Pi_{\mathcal{S}}$ is a POVM. This also gives

$$
\sum_{s} p_{s}=1
$$

for the probabilities for $s$ to occur.

The Holevo quantity for the communication of (classical) information about $s$ is given by

$$
\chi\left(\Pi_{\mathcal{S}}: \mathcal{F}\right)=H\left(\rho_{\mathcal{F}}\right)-\sum_{s} p_{s} H_{\mathcal{F} \mid s},
$$

where we used Eq. (23). Taking Eq. (12) and the assumption that for the pointer basis the classical information is at the plateau value, i.e., that there is a perfect record, one obtains

$$
\begin{aligned}
\chi\left(\Pi_{\mathcal{S}}: \mathcal{F}\right)= & H\left(\sum_{\hat{s}} p_{\hat{s}} \rho_{\mathcal{F} \mid \hat{s}}\right) \\
& -\sum_{s} p_{s} H\left(\sum_{\hat{s}} p_{s \hat{s}} p_{\hat{s}} \rho_{\mathcal{F} \mid \hat{s}} / p_{s}\right) \\
= & H\left(p_{\hat{s}}\right)+\sum_{\hat{s}} p_{\hat{s}} H_{\mathcal{F} \mid \hat{s}} \\
& -\sum_{s} p_{s}\left[H\left(p_{s s} p_{\hat{s}} / p_{s}\right)+\sum_{\hat{s}} p_{s \hat{s}} p_{\hat{s}} / p_{s} H_{\mathcal{F} \mid \hat{s}}\right] \\
= & H\left(p_{\hat{s}}\right)+\sum_{\hat{s}} p_{\hat{s}} H_{\mathcal{F} \mid \hat{s}} \\
& -\sum_{s} p_{s} H\left(p_{s \hat{s}} p_{\hat{s}} / p_{s}\right)-\sum_{\hat{s}} p_{\hat{s}} H_{\mathcal{F} \mid \hat{s}} \\
= & H\left(p_{\hat{s}}\right)+\sum_{s, \hat{s}} p_{s \hat{s}} p_{\hat{s}} \log \left(p_{s \hat{s}} p_{\hat{s}} / p_{s}\right) \\
= & H\left(\Pi_{\mathcal{S}}\right)-H\left(\Pi_{\mathcal{S}} \mid \hat{\Pi}_{\mathcal{S}}\right)
\end{aligned}
$$

for the plateau value of the classical information about $s$ deposited in the environment: Its value is suppressed by the conditional entropy of $\Pi_{\mathcal{S}}$ with respect to the pointer basis $\hat{\Pi}_{\mathcal{S}}$, i.e., the misalignment of $\Pi_{\mathcal{S}}$ from $\hat{\Pi}_{\mathcal{S}}$.

The presence of redundant information requires that

$$
\chi\left(\Pi_{\mathcal{S}}: \mathcal{F}\right) \geq H\left(\Pi_{\mathcal{S}}\right)(1-\delta),
$$

where $H\left(\Pi_{\mathcal{S}}\right)$ measures the amount of missing information. This can not be satisfied when

$$
H\left(\Pi_{\mathcal{S}} \mid \hat{\Pi}_{\mathcal{S}}\right)>\delta \cdot H\left(\Pi_{\mathcal{S}}\right)
$$

which makes it clear that when the POVM is rotated away from the pointer basis, eventually redundant information cannot be present in the environment.

Furthermore, even for an environment with an infinite number of components, whether or not there is redundant information about the POVM depends on the accuracy required: All POVMs that are not the pointer basis will not be redundantly encoded if one requires a small information deficit. In this sense, we say that only pointer states can be found out.

Of course, one can also start from the expression Eq. (14) for branched states and use the conservation law to get

$$
\chi\left(\Pi_{\mathcal{S}}: \mathcal{F}\right)=\chi\left(\hat{\Pi}_{\mathcal{S}}: \mathcal{F}\right)-\sum_{s} p_{s} H_{\mathcal{F} \mid s}
$$

Thus, the Holevo quantity will be reduced for all POVMs that are not the pointer basis. As the generation of the branched state gives rise to surplus decoherence and the fragment starts to acquire a perfect record, Eq. (38) will approach Eq. (35).

We can quantify this approach for imperfect records. We assume surplus decoherence and that the pointer states are correlated with nearly distinguishable states $\rho_{\mathcal{F |} \mid \hat{s}}$, i.e., that one is very near the classical plateau. The latter implies that there exists a POVM $\Lambda$ on $\mathcal{F}$, with elements $\Lambda_{\hat{s}}$, such that $\operatorname{tr} \Lambda_{\hat{s}} \rho_{\mathcal{F} \mid \hat{s}} \geq 1-\epsilon$ and $\operatorname{tr} \Lambda_{\hat{s}^{\prime}} \rho_{\mathcal{F} \mid \hat{s}} \leq \epsilon$ for $\hat{s}^{\prime} \neq \hat{s}$, where $\epsilon$ gives the error probability for distinguishing the states. Let the measurement be carried out by apparatus $\mathcal{A}$. We can apply this to studying the Holevo quantity with respect to some arbitrary POVM on $\mathcal{S}$ :

$$
\chi\left(\Pi_{\mathcal{S}}: \mathcal{F}\right)=\chi\left(\Pi_{\mathcal{S}}: \mathcal{F} \mathcal{A}\right)_{\rho}=\chi\left(\Pi_{\mathcal{S}}: \mathcal{F} \mathcal{A}\right)_{\tilde{\rho}}
$$

where first $\rho_{\mathcal{S F}} \rightarrow \rho_{\mathcal{S F} \mathcal{A}}=\rho_{\mathcal{S F}} \otimes|0\rangle_{\mathcal{A}}\langle 0|$ and then a unitary acts on $\mathcal{F} \mathcal{A}$, with its relevant action defined by $\mathcal{U}_{\mathcal{F A}}|\psi\rangle_{\mathcal{F}}|0\rangle_{\mathcal{A}}=\sum_{\hat{s}} \sqrt{\Lambda_{\hat{s}}}|\psi\rangle_{\mathcal{F}}|\hat{s}\rangle_{\mathcal{A}}$, to get $\tilde{\rho}_{\mathcal{S F} \mathcal{A}}$, neither of which change the Holevo quantity. Then, however, the positive operator $\Omega=\sum_{\hat{s}}|\hat{s}\rangle\left\langle\hat{s}\left|\otimes \mathbf{I}_{\mathcal{F}} \otimes\right| \hat{s}\right\rangle_{\mathcal{A}}\langle\hat{s}| \leq \mathbf{I}$ has the property 


$$
\operatorname{tr} \Omega \tilde{\rho}_{\mathcal{S F A}} \geq 1-\epsilon
$$

Thus, the state

$$
\sigma_{\mathcal{S F A}}=\frac{\sqrt{\Omega} \tilde{\rho}_{\mathcal{S F A}} \sqrt{\Omega}}{\operatorname{tr} \Omega \tilde{\rho}_{\mathcal{S F A}}}
$$

has

$$
\frac{1}{2} \operatorname{tr}\left|\sigma_{\mathcal{S F A}}-\tilde{\rho}_{\mathcal{S F} \mathcal{A}}\right| \leq \sqrt{\epsilon}
$$

by the gentle measurement lemma ${ }^{43,44}$. The analysis leading to Eq. (35) holds as $\mathcal{S}$ and $\mathcal{A}$ are perfectly correlated in the state $\sigma_{\mathcal{S F} \mathcal{A}}$. We can then apply the Alicki-Fannes' inequality $^{45}$

$$
\begin{gathered}
\left|\chi\left(\Pi_{\mathcal{S}}: \mathcal{F}\right)-\left(H\left(\Pi_{\mathcal{S}}\right)-H\left(\Pi_{\mathcal{S}}\left|\hat{\Pi}_{\mathcal{S}}\right|\right)\right)\right| \\
=\left|\chi\left(\Pi_{\mathcal{S}}: \mathcal{F} \mathcal{A}\right)_{\tilde{\rho}}-\chi\left(\Pi_{\mathcal{S}}: \mathcal{F} \mathcal{A}\right)_{\sigma}\right| \\
=\left|H\left(\Pi_{\mathcal{S}} \mid \mathcal{F} \mathcal{A}\right)_{\tilde{\rho}}-H\left(\Pi_{\mathcal{S}} \mid \mathcal{F} \mathcal{A}\right)_{\sigma}\right| \\
\leq 8 \sqrt{\epsilon} \log D_{\Pi}+2 H(2 \sqrt{\epsilon}),
\end{gathered}
$$

where $D_{\Pi}$ is the number of outcomes for the POVM $\Pi_{\mathcal{S}}$. Thus, the reduction in the classical information about non-pointer $\Pi_{\mathcal{S}}$ is due to $H\left(\Pi_{\mathcal{S}} \mid \hat{\Pi}_{\mathcal{S}}\right)$ up to small corrections. For the case of branching states, these corrections are exponentially small in the size of the fragment.

The surplus decoherence assumption can be similarly relaxed, i.e., by assuming $\rho_{\mathcal{S F}}$ is near to the discord-free state. For branching states, $\rho_{\mathcal{S F}}$ is exponentially close in the size of environment to a discord free state. Thus, the $\epsilon$ above, while small, dominates how close the exact $\chi\left(\Pi_{\mathcal{S}}: \mathcal{F}\right)$ is to the perfect record case. The case of imperfect records but mixed states extends the results of Ref. 12 .

1. Bohr, N. The quantum postulate and the recent development of atomic theory Nature 121, 580-590 (1928).

2. Einstein, A., Podolsky, B. \& Rosen, N. Can quantum-mechanical description of physical reality be considered complete? Phys. Rev. 47, 777-780 (1935).

3. Bohr, N. Can quantum-mechanical description of physical reality be considered complete? Phys. Rev. 48, 696-702 (1935).

4. Schrödinger, E. Die gegenwärtige situation in der quantenmechanik. Naturwissenschaften 23, 807-812 (1935).

5. Bohr, N. Atomic Physics and Human Knowledge (Wiley, New York, 1958).

6. Joos, E. et al. Decoherence and the Appearance of a Classical World in Quantum Theory (Springer-Verlag, Berlin, 2003).

7. Zurek, W. H. Decoherence, einselection, and the quantum origins of the classical. Rev. Mod. Phys. 75, 715-775 (2003).

8. Schlosshauer, M. Decoherence and the Quantum-to-Classical Transition (Springer-Verlag, Berlin, 2008).

9. Zurek, W. H. Pointer basis of quantum apparatus: Into what mixture does the wave packet collapse? Phys. Rev. D 24, 1516 (1981)

10. Zurek, W. H. Environment-induced superselection rules. Phys. Rev. D 26, 1862 (1982).

11. Ollivier, H., Poulin, D. \& Zurek, W. H. Objective properties from subjective quantum states: Environment as a witness. Phys. Rev. Lett. 93, 220401 (2004).

12. Ollivier, H., Poulin, D. \& Zurek, W. H. Environment as a witness: Selective proliferation of information and emergence of objectivity in a quantum universe. Phys. Rev. A 72, 042113 (2005).

13. Blume-Kohout, R. \& Zurek, W. H. A simple example of quantum Darwinism: Redundant information storage in many-spin environments. Found. Phys. 35, $1857-1876$ (2005).

14. Blume-Kohout, R. \& Zurek, W. H. Quantum Darwinism: Entanglement, branches, and the emergent classicality of redundantly stored quantum information. Phys. Rev. A 73, 062310 (2006).

15. Blume-Kohout, R. \& Zurek, W. H. Quantum Darwinism in quantum brownian motion. Phys. Rev. Lett. 101, 240405 (2008).

16. Bennett, C. H. The second law and quantum physics. AIP Conf. Proc. 1033, 66-79 (2008).

17. Brunner, R., Akis, R., Ferry, D. K., Kuchar, F. \& Meisels, R. Coupling-induced bipartite pointer states in arrays of electron billiards: Quantum Darwinism in action? Phys. Rev. Lett. 101, 024102 (2008).

18. Zwolak, M., Quan, H. T. \& Zurek, W. H. Quantum Darwinism in a mixed environment. Phys. Rev. Lett. 103, 110402 (2009).

19. Zurek, W. H. Quantum Darwinism. Nat. Phys. 5, 181-188 (2009).

20. Paz, J. P. \& Roncaglia, A. J. Redundancy of classical and quantum correlations during decoherence. Phys. Rev. A 80, 042111-042116 (2009).

21. Zwolak, M., Quan, H. T. \& Zurek, W. H. Redundant imprinting of information in nonideal environments: Objective reality via a noisy channel. Phys. Rev. A 81, 062110 (2010).
22. Riedel, C. J. \& Zurek, W. H. Quantum Darwinism in an everyday environment: Huge redundancy in scattered photons. Phys. Rev. Lett. 105, 020404 (2010).

23. Burke, A. M. et al. Periodic scarred states in open quantum dots as evidence of quantum Darwinism. Phys. Rev. Lett. 104, 176801 (2010).

24. Riedel, C. J. \& Zurek, W. H. Redundant information from thermal illumination: Quantum Darwinism in scattered photons. New Journal of Physics 13, 073038 (2011).

25. Riedel, C. J., Zurek, W. H. \& Zwolak, M. The rise and fall of redundancy in decoherence and quantum darwinism. New Journal of Physics 14, 083010 (2012).

26. Holevo, A. S. Bounds for the quantity of information transmitted by a quantum communication channel. Probl. Peredachi Inf. 9, 3-11 (1973).

27. Zurek, W. H. Einselection and decoherence from an information theory perspective. Ann. Phys.-Leipzig 9, 855-864 (2000).

28. Ollivier, H. \& Zurek, W. H. Quantum discord: A measure of the quantumness of correlations. Phys. Rev. Lett. 88, 017901 (2002).

29. Henderson, L. \& Vedral, V. Classical, quantum and total correlations. J. Phys. A: Math. Gen. 34, 6899 (2001).

30. Groisman, B., Popescu, S. \& Winter, A. Quantum, classical, and total amount of correlations in a quantum state. Phys. Rev. A 72, 032317 (2005).

31. Zurek, W. H., Habib, S. \& Paz, J. P. Coherent states via decoherence. Phys. Rev. Lett. 70, 1187-1190 (1993).

32. Zurek, W. H. Preferred states, predictability, classicality and the environmentinduced decoherence. Prog. Theor. Phys. 89, 281 (1993).

33. Paz, J. P. \& Zurek, W. H. Environment-induced decoherence and the transition from quantum to classical. 72nd Les Houches Summer School on "Coherent Matter Waves", arXiv:quant-ph/0010011 (2000).

34. Cover, T. M. \& Thomas, J. A. Elements of information theory (Wiley-Interscience, New York, 2006).

35. Datta, A. Studies on the role of entanglement in mixed-state quantum computation. Ph. D. Thesis, arXiv::0807.4490 (2008).

36. Greenberger, D. \& YaSin, A. "Haunted" measurements in quantum theory. Found. Phys. 19, 679-704 (1989).

37. Carmichael, H. J., Kochan, P. \& Tian, L. Coherent states and open quantum systems: A comment on the Stern-Gerlach experiment and Schrödinger's cat. In Feng, D., Klauder, J. R. \& Strayer, M. R. (eds.) Proceedings of the International Symposium on Coherent States, Past, Present and Future 75-91 (World Scientific, Singapore, 1994).

38. Wootters, W. K. \& Zurek, W. H. A single quantum cannot be cloned. Nature $\mathbf{2 9 9}$, 802-803 (1982).

39. Dieks, D. Communication by EPR devices. Physics Letters 92A, 271-272 (1982).

40. Everett, H. "Relative state" formulation of quantum mechanics. Rev. Mod. Phys. 29, 454 (1957).

41. Streltsov, A. \& Zurek, W. H. Quantum discord cannot be shared, arXiv:1304. 2914 (2013).

42. Nielsen, M. A. \& Chuang, I. L. Quantum Computation and Quantum Information (Cambridge University Press, Cambridge, UK, 2000).

43. Winter, A. Coding theorem and strong converse for quantum channels. Information Theory, IEEE Transactions on 45, 2481-2485 (1999).

44. Ogawa, T. \& Nagaoka, H. Making good codes for classical-quantum channel coding via quantum hypothesis testing. Information Theory, IEEE Transactions on 53, 2261-2266 (2007).

45. Alicki, R. \& Fannes, M. Continuity of quantum conditional information. J. Phys. A: Math. Gen. 37, L55 (2004)

\section{Acknowledgments}

This research is supported in part by the U.S. Department of Energy through the LANL/ LDRD Program and in part by the John Templeton Foundation. We would also like to thank the Center for Integrated Quantum Science and Technology (IQST), Universität Ulm, where part of this work was carried out.

\section{Author contributions}

M.Z. and W.H.Z. both contributed to this research and the writing of the manuscript.

\section{Additional information}

Supplementary information accompanies this paper at http://www.nature.com/ scientificreports

Competing financial interests: The authors declare no competing financial interests.

License: This work is licensed under a Creative Commons

Attribution-NonCommercial-NoDerivs 3.0 Unported License. To view a copy of this license, visit http://creativecommons.org/licenses/by-nc-nd/3.0/

How to cite this article: Zwolak, M. \& Zurek, W.H. Complementarity of quantum discord and classically accessible information. Sci. Rep. 3, 1729; DOI:10.1038/srep01729 (2013) 